\title{
VII. Ein wiedererstandenes Klosterarchiv in Steiermark.
}

\author{
Von \\ P. Jacob Wichner, \\ Stiftsarchivar und Bibliothekar zu Admont.
}

Während so viele Klöster, mit denen Oestreichs lachende Fluren ehemals besäet waren, den Anschauungen und Stürmen der neuen Zeit zum Opfer fielen - in Steiermark waren es mehr als dreissig an del Zahl - hatten einige, sei es in Würdigung ihrer Leistungen oder durch Verwendung māchtiger Gönner, Gnade gefunden und die Aufhebungsperiode überlebt. $\mathrm{Zu}$ diesen zählt die Benedictinerabtei Admont an der Enns im steirischen Oberlande. Schon früher nicht unbekannt wurde der Ort seit der Eröffnung der Kronprinz-Rudolph-Bahn Zielpunkt zahlreicher Ausflüge. Und in der That, wenn nicht schon das Stift mit seiner herrlichen Bibliothek und seinem gothischen Dome eines Besuches werth wäre, würde die Natur, welche alle ihre Reize über das Thal verschwenderisch ausgegossen hat, eine zauberische Gewalt ausüben. Eine andere Lorelei scheint sich in diesem Thale ihr Heim gesucht zu haben, und aus jeder Felsenspalte, aus jedem Waldesdickicht blickt ihr lockendes Antlitz.

Doch hier reden wir nicht von landschaftlichen Schönheiten, sondern nur vom Admonter Archiv, und da ich mich als Führer durch dasselbe anbiete, muss ich mich wohl selbst erst vorstellen. Da komme ich mir in Gesellschaft von Historikern und Archivaren vor, wie Saul unter den Propheten. Mein Bewusstsein sagt mir: $\gg$ Du bist kein eigentlicher Fachmann; dir fehlen alle diplomatischen Vorstudien; du hast ja erst, als der erste Schnee auf deinem Haupte sich zu lagern begann, mit schüchterner Hand den Vorhang zu 
lüften versucht, der die Pforte zum Heiligthum der Wissenschaft verbirgt. Wenn ich aber bedenke, dass bei einem Monumentalbaue nicht nur Meister und Künstler, sondern auch untergeordnete Werkleute sich thätig erweisen, so erscheint auch derjenige, welcher zum Tempelbaue historischer Forschung brauchbare Bausteine sammelt, zurechtlegt und hier und da einfügt, auf freundlichen Empfang rechnen zu dürfen.

Der Stand, welchen ich mit freier Wahl ergriff, war der geistliche im Orden des $h$. Benedict, und die mir von meinen Oberen zugewiesene Wirkenssphäre war die Seelsorge, in welcher ich mehr als 18 Jahre, mitunter in den abgelegensten Gebirgsschluchten, zubrachte. Wohl fühlte ich mich schon frühzeitig angeregt, die Geschichte meines engeren Vaterlandes Steiermark kennen zu lernen, und als ich das Ordenskleid angezogen hatte, machte ich meine ersten historischen Versuche, indem ich Chroniken von Admonter Pfarren verfasste. Den Stoff schöpfte ich dazu meist aus Druckwerken. An Quellen waren mir nur die Pfarrmatrikeln und einzelne Urkunden und Akten zugänglich. Wohl wusste ich um die Existenz eines Stiftsarchives zu Admont, allein es gab für einen Landkaplan, von dem man in damaliger Zeit kein ernstliches historisches Forschen erwartete, der Schwierigkeiten zu viele, welche sich der Benützung des Archives entgegenstellten. Als ich im Winter 1859-1860 einige Monate zu Admont zubrachie, betrat ich einige Male das Archiv, an der Hand des strengen und sehr zugeknöpften Archivars, um einige Bānde der Archidiakonatsakten in Empfang zu nehmen oder zurūck zu bringen. Der Aufenthalt in diesem ehrwürdigen Raume dauerte jedesmal nur einige Minuten, und vor meiner Erinnerung schweben bloss noch bestaubte Aktenbündel und Bullen und Siegel, die man nachlässig hier und dort herabhängen liess.

Damals wurde mir freigestellt, auch die Ordnung des Archives zu übernehmen, allein ich sollte auch Kanzleidirektor werden. Dazu fehlte es mil an Lust und überdies würde es mir schwer gefallen sein, der Seelsorge Valet zu sagen. Ich machte einen Gegenvorschlag, man möge mir die Leitung des Archives und der Bibliothek übertragen. Ich war und bin der Ansicht, dass beide Institute sich gegenseitig ergänzen, und dass das Wirken eines Archivars sich fruchtbringender gestaltet, wenn ihm die reichen Hilfsmittel der Bibliothek ohne Beschränkung jeder Zeit zu Gebote stehen. Auch sind in Admont in den Handschriften und auf den Innendeckeln 
der Incunabeln der Stiftsbibliothek zahlreiche Lrkunden eingeklebt und historische Notizen eingetragen. Viele solcher Handschriften hätte man ihrem Inhalte nach in's Archiv einreihen müssen. Mein Antrag sollte sich aher erst in diesem Jahre 1878 erfüllen: achtzehn Jahre früher wurde er abgelehnt, und ich ging auf's Land zurück. Im Jahre 18.0 aber, als ich Pfarrer zu Ardning war, überfiel nich ein Herz- und Nervenleiden, welches mich für die Anstrengungen der Seelsorge unfähig und die Rückkehr in das Stift zur unabweisharen Nothwendigkeit machte. Hier hatte sich aber Vieles geändert. Der schreckliche Brand vom 27. April 1865 hatte nicht nur einen Theil des Marktfleckens vernichtet, auch das Stiftsgebäude sammt der Kirche, die naturhistorischen und Kunstsammlungen (mit Ausnahme der Bibliothek) waren eine Beute des wüthenden Elementes geworden. Das Hauptarchiv ging zu Grunde, ohne dass das Geringste davon geborgen werden konnte. Dasselbe fing erst am zweiten Tage zu brennen an; entweder hatte man bei dem grossen Unglücke den Kopf verloren, oder im Vertrauen auf die Feuersicherheit des Lokales sich nicht weiter um das Archiv bekümmert das Unheil war nun einmal geschehen. Dass dieser Verlust von mir und meinen Mitbrüdern tief bedauert wurde, bedarf keiner Versicherung.

Nur ein Trost war uns geblieben. Was man sonst als unzweckmässig anerkennt, nämlich die Zersplitterung der Archivalien in verschiedenen von einander entlegenen Räumen, war in unserem Falle ein Glück zu nennen. Wurde doch dadurch wenigstens ein Theil des werthvollen Schatzes erhalten und konnte als Grundstock bei Organisirung eines neuen Archives verwerthet werden. Es bestanden nāmlich neben dem abgebrannten Hauptarchive zwei kleinere, das Prälatur- oder Kapellenarchiv und das Thurmarchiv. Wir werden auf Beide zurückkommen, und möge hier eine gedrängte Uebersicht der Entwicklung des admontischen Archivwesens vorausgeschickt werden.

Es war natürlich, dass bei einer Körperschaft, deren Wirksamkeit sich nicht auf die engen Thalmarken der Enns beschränkte, sondern deren Grund- und Gültenbesitz, durch Schenkang und Ankauf bis in das 16. Jahrhundert stätig vermehrt, über ganz Steiermark, auf Kärnten, Ober- und Niederöstreich, Salzburg, Tirol, Friaul und Bayern sich ausdehnte, im Laufe der Zeit eine Menge von Urkunden und Akten sich anhäufen musste. In den ersten 
Jahrhunderten nach Gründung der Abtei, welche 1074 erfolgte, wurden die schriftlichen Rechtsbehelfe neben den Kirchenkleinoden im Sagrer (Sakristei) hinterlegt und waren der Obhut des Gusters (custos) anvertraut. Für Admont haben wir darüber einen directen historischen Beleg. Als Erzbischof Eberhard II. von Salzburg 1202 die Schenkung der Pfarre Jahring an das Stift erneuerte, erwāhnt er ausdrücklich, dass das Original des ersten Donationsbriefes im Sacrarium des Blasienmünsters aufbehalten sei. •Exemplum quiden actionis hujus seu traditionis a domino Chunrado seniore archiepiscopo quondam factae chyrographum ipsius praesulis sigillo munitum et in sacrario praefati monasterii repositum.\& Darin findet auch die Thatsache Erklärung, dass häufig, anfangs sogar in der Regel, der Guster, als Hüter der Dokunente, die Gerechtsame des Klosters vor der Gerichtsschranne vertreten musste. Aber auch die sorgfältigste Verwahrung der Membrane konnte nicht empfindlichen Verlusten vorbeugen und das Heiligthum der Kirche war keine Schutzwelr gegen List und Gewalt. So ist die Stiftungsurkunde Admonts bei den wiederholten Einfallen des Aftererzbischofs Berthold von Moosburg und des gewaltthätigen Grafen Adalbero des Rauhen zwischen 1078 und 1090 verloren gegangen ${ }^{1}$ ).

Schon im 14. Jahrhundert beginnen auf der Rückseite admontischer Urkunden Signaturen in rother Farbe (grosse Buchstaben und römische Ziffern); bei einigen findet sich das Regest verzeichnel, z. B. „ Vmb ein chrieg zwischen Alrams ouz dem Steinach vnd dez gotshaus«, ein Beweis, dass eine systematische Beschreibung und Einreihung der Pergamente Platz gegriffen hatte. Wahrscheinlich war dieser erste Ordnungsplan ein Verdienst des Abtes Ekhard (132 bis 1338), aus dessen Regierungszeit auch ein noch vorhandenes Urbar herstammt. Im 15. und 16. Jahrhundert pflegten die Aebte selbst, oder deren Anwälte und Sekretäre die Aufsicht über die Archivalien $\mathrm{zu}$ handhaben, welche unter dem Abte Valentin (1545 bis 1568) in "Truhen " verschlossen waren. Abt Mathias Preininger (1615 bis 1628), welcher Kirche und Klostergebäude neu herstellte,

1) Dass das heim Stiftshrande abhanden gekommene und bislang als Stifthrief angesehene Dokument nur ein um 1106 zusammengestelltes und vom Erzhischof Conrad I. gesiegeltes Gũterverzeichniss gewesen sei, habe ich in meiner sGeschichte d. Bened. Stiftes Admont . . . 1. 32. 38. 233 nachgewiesen. Auch Zahn , Urkundenbuch des Herzogthums Steiermark I. 95 bekennt sich zur gleichen Ansicht. 
liess im nördlichen Yünsterthurme ein Lokal für das Archiv einrichten. Im Jahre $1659 \mathrm{nahm} \mathrm{P.} \mathrm{Christof} \mathrm{Schnuek} 744$ der wichtigsten Urkunden in ein von ihm verfasstes Copialbuch auf. Abt Adalbert Heufler von Rasen liess 1690 durch den Conventualen Anselm Luerzer ein Inventar des Archivs aufnehmen, dabei klebte Dieser kleine Zettel mit neuen Signaturen und Regesten auf die Urkunden. Damals war es auch, dass ein Theil des Archivbestandes, besonders Archidiakonats- und Pfarrakten ${ }^{1}$ ), in die Prälatur übertragen wurde, wo er das Kapellenarchiv bildete. Als Archivare sind ausser Schmuek und Luerzer noch bekannt: Michael Seiz (1751), Isingrin Haslinger (1785) und Edmund Prandstetter († 1810). Unter dem Abte Benno (1823-1861) waren Bibliothek und Archiv unter einer Leitung. Zwei der verdienstvollsten Männer, Albert von Muchar und Urban Ecker, standen beiden Instituten vor. Muchars Name hat guten Klang in den Kreisen der Historiker; Ecker glänzte nicht vor der Welt, aber er hat vielseitige Spuren seines Fleisses und reichen Wissens hinterlassen. Vor dem Stiftsbrande bekleidele P. Barnabas Mauer die Stelle eines Archivars.

Ueberhaupt hat es in der Admonter Brüdergemeinde nie an Iaannern gefehlt, welche der historischen Wissenschaft einen Theil ihrer Kräfte zuwandten und mit Vorliebe das Feld der Hausgeschichte bearbeiteten. Das Chronicon Admontense (Codex 501 der Bibliothek), die zwei Codices praediorum oder Grundbücher, die Libri traditionum oder Salbücher II., III. und IV. dem 12. und 13. Jahrhundert angehörig, haben einheimische Verfasser.

Das Salbuch I. enthielt die Series abbatum und eine Stiftsgeschichte und wurde um 1580 von dem Admonter Mönche -.Theodosius Lang geschrieben. Alle diese Handschriften sind mit Ausnahme des Codex 501 verbrannt. Die Bibliothekshandschrift 475 (saec. XIII.) enthält zahlreiche Urkundenabschriften. Aus dem 15. Jahrhundert ist ein metrischer Aebtekatalog zu nennen, welcher mit dem Tode des Abtes Hartnid (1413) abschliesst. P. Modest Puterer ( $†$ 1559) schrieb eine kurzgefasste Ordensgeschichte. Annalen des Stiftes wurden von Benedikt Hammerschall $(\dagger 165 \%$, Michael Seiz ( $\dagger$ 1714) und Bonifaz Kuchler ( $\dagger$ 1744) hinterlassen. Von Carlmann Vierholz stammt ein Aufsatz »Ortus et progressus monasterii Admontensis«.

1) Von 1648 bis 1784 fungirten die Aebte von Admont als Arcbidiakone im Enns-, Palten- und Liesingthale. 
In neuerer Zeit haben Albert ron Muchar, Dr. Richard Peinlich, Dr. Gregor Fuchs und Florian Kinnast Erhebliches für die Geschichte des Landes und des Stiftes geleistet.

Den Inhalt des Hauptarchivs vor dem Brande auch nur in allgemeinen Umrissen zu schildern, hat seine Schwierigkeit, weil auch alle Repertorien und Inventare des Urkunden-, Handschriftenund Aktenbestandes in Verlust gerathen sind. Soviel steht aber fest, dass das Hauptarchiv, was den Werth und die Fülle des Materiales betrifft, manches grosse Landesarchiv übertroffen hat. Die ālteste Urkunde dürfte das Confirmationsdiplom des Papstes Pascal II. von 1105 gewesen sein. Ich will es rersuchen, auf Grundlage verlässlicher Aufzeichnungen die wichtigsten Urkunden des 12. und 13. Jahrhunderts zu verzeichnen. An Bestātigungs- und Gnadenbriefen waren vorhanden jene von Innocenz II. (1139), Lucius II. (1144), Alexander III. (1171), Lucius III. (1185), Urban III. (1187), Innocenz IV. (1247). (Ausserdem noch sieben Urkunden): von Innocenz III. (1210), Gregor IX. (1230), Alexander IV. (1255, 2 Urk.), Urban IV. (1262), Clemens IV. (1265, 5 Urk.), Nicolaus IV. (1291, 2 Urk.), Majestätsdiplome der Kaiser und Könige von Friedrich I. (1184. 1189), Heinrich VI. (1194), Otto IV. (1209), Friedrich II. (1135), Rudolf I. (1276. 1281. 1290), Ottocar von Böhmen (1267. 1270). Die Traungauer fanden ihre Vertretung durch Herzog Ottocar VIII. (circa 1180. 1186), die Babenberger durch Heinrich II. (1169), Leopold den Glorreichen (1196. 1227), und Friedrich den Streitbaren (1242), und die habsburgischen Herzoge durch Albrecht I. (1283. 1284. 1290). Wenn wir diesen erlauchten Namen noch Salzburgs Oberhirten Conrad I., II. und IV., Eberhard I. und II., Adalbert III.. Friedrich II. und Rudolf, die Grafen von Görz, Andechs, Wasserburg und Abensberg anreihen, lässt sich der Werth des leider unwiederbringlich Verlorenen ermessen.

Die Aebtesiegel reichten bis zum Abte Jsinrik (1186) zurück, welcher Zeit auch die erste von einem Admonterabte ausgestellte (noch vor dem Brande erhaltene) Urkunde angehörte. Frühere Aebteurkunden, obwohl nachweisbar ausgestellt, waren schon lăngst dem Zahne der Zeit verfallen.

An eigentlichen Handschriften sind ausser den schon genannten Sal- und Grundbüchern und den Chroniken die zwei älteren Todtenbücher (Signatur C 543 und 544), dem 12. und 13. Jahrhundert zugehörig, und ein Directorium antiquissimum Admontense (C 381) 
als verloren zu beklagen. Aus den zwei Nekrologien hat $\mathrm{Pez}$ Scriptores II. 199-209 Excerpte ${ }^{\circ}$ geliefert. Das Directorium habe ich in meiner Stiftsgeschichte II. 186-18\%, 229-238 nach Muchars Abschrift wieder gegeben.

An Kauf- und Schirmbriefen (16.-19. Jahrhundert) waren bei 25,000 Stücke aufgespeichert.

Wie in allen Archiven haben die Akten den Löwenantheil des Raumes in Anspruch genommen. Sie bestanden in Processen, Rechnungen, Correspondenzen, Kauf- und Verlasshandlungen, Inventaren, Archidiakonats- und Pfarrakten. Wieviel des Interessanten, wie manches archivalische Unicum mag das Hauptarchiv geborgen haben, allein die grausame Flamme hat selbst die Kenntniss des Vorhandenseins vertilgt.

Ein grosser Theil des Thurmarchives war vor dem Brande dem Hauptarchive einverleibt worden, allein gewiss nicht Alles. Als der Neubau der Kirche und des Klosters begann, haben die Arbeiter und wohl auch Andere noch Vieles verschleppt und zu Zwecken benützt, welche historischer Forschung ferne liegen. Ich selbst fand, als ich 1870 das Lokal, welches durch den Orgelkasten seinen Zugang hatte, besuchte, die Repositorien gewaltsam gesprengt, und viele Akten des 16. Jahrhunderts im Staube des Fussbodens liegen. Ein Convolut Akten übergab mir Hr. Oberlehrer Karl Mayr. Auch zu Admont waren sunbrauchbare Pergamente zum Einbinden von Büchern, zum Verkleben des Blasebalges der Orgel und zu andern »technischen « Zwecken verwendet worden. Im Uebrigen fehlen über den ehemaligen Inhalt des Thurmarchives alle Anhaltspunkte. Das Prälatur- oder Kapellenarchiv aber, welches der Katastrophe entgangen ist, bildet den Grundstock des wieder erstandenen Admonter Archives, welches zu schildern meine Aufgabe sein wird. Auch dem Prälaturarchive hatte der Untergang gedroht, denn einzelne Fascikel tragen Brandspuren und an nicht wenigen Urkunden sind die Siegel zerschmolzen.

Ich kam, wie erwähnt, im Sommer 1870 wieder in das Stift zurück. Meine erste Sorge war es, mir einen neuen meinen Kräften entsprechenden und zugleich meinem geistlichen Vaterhause erspriesslichen Wirkungskreis zu erobern. Von meinem Mitbruder, dem leider zu früh gestorbenen Naturhistoriker und Verfasser einer Topographie des Admontthales P. Thassilo Weymayr, erfuhr ich, dass noch »einige Urkunden und Schriften dem Verderben entronnen seien. 
Der Zufall, oft der Vater grosser Entdeckungen, war meinen Bestrebungen hold. Im Herbste desselben Jahres traf es sich, dass der steiermärkische Landesarchivar, Hr. Prof. Josef v. Zahn, dem ich vielseitige Anregung und Unterstützung verdanke, Admont berührte, um für das steiermärkische Urkundenbuch und das Landesarchiv Urkunden oder Abschriften zu erwerben. Bei dieser Gelegenheit fügte es sich, dass ich mit ihm und P. Florian Kinnast, Verfasser des Album Admontense und mehrerer historischen Aufsätze, zum ersten Male jenen Ort betrat, welcher den geretteten Archivresten als Zufluchtsstātte diente. Aber in welchem Zustande fand ich das ersebnte Heiligthum! Im zweiten Stocke des Südtractes lag ein saalähnliches Zimmer, von sechs Fenstern erhellt, längs dessen Wänden 16 Schränke verschiedener Form und Grösse aufgestellt waren. Die meisten waren versperrt, und auf meine Anfrage nach deren Inhalt wurde mir bedeutet, dass sie - leer seien. Die nicht verschlossenen hatten lange und tiefe Schubladen, welche mehr oder minder mit Akten und Dokumenten gefüllt waren. Das Ganze starrte von Staub und Spinnengeweben, altes Gerümpel stand und lag umher, und was dem Gräuel die Krone aufsetzte, war, dass der Stiftsapotheker hier sein Kräutermagazin etablirt hatte. Dass die Versuchung nahe lag und derselben auch nachgegeben worden war, die »unnützen. Papiere als enveloppes für die Produkte Aesculaps und Hygieias zu verwerthen, davon überzeugte mich spätere Nachforschung. Unsere erste Entdeckungsreise sollte aber nicht resultatlos bleiben. Einige oberflächlich in das Staubmeer gemachte Griffe brachten bei 8 Stück Urkunden des 12. und 13. Jahrh. zum Vorschein.

Nachdem ich einmal jene Stelle entdeckt hatte, wo der archivalische Schatz der Hebung harrte, fand ich mich erst beruhigt, als mir die Vollmacht gegeben wurde, Licht in das Chaos zu bringen und nach Möglichkeit das Archiv neu zu gestalten. Der Apotheker bekam die Weisung, sein Herbar an einen andern Ort zu verlegen. Doch Tag für Tag, Woche für Woche floss dahin, die Kräuter behaupteten ihren Lagerplatz. Da hatte meine Geduld ein Ende. Eines schönen Morgens warf ich die vertrockneten Kinder Floras, wohl nicht in der besten Ordnung, auf den Corridor hinaus. Ein Feind war siegreich bekämpft. Nun wurde der Feldzug gegen Măuse, Spinnen und Staub organisirt und glücklich durchgeführt. Endlich konnte ich frei und froh auf dem mühselig errungenen Felde schalten und walten. Lade um Lade wurde herausgezogen, und wenn sich 
unter dem Wuste alter Rechnungen ein Schriftstück des 15. Jahrh. oder ein rergilbtes Pergament fand, so pochte mein Herz in freudiger Stimmung. Ueber ein kleines Kistchen mit Glasscherben gefüllt war ich mehrmals gestolpert; schon war ich im Begriffe, dasselbe aus dem Lokal zu entfernen, als mein guter Genius rieth, näher nachzusehen. Nur ein Archivar kann die Freude würdigen, die ich empfand, als ich unter den Glastrümmern bei 60 Stūck Originalurkunden des 13. und 14. Jahrh. hervorlangen konnte. Und als ich die oben erwähnten rersperrten Schränke öffnete, sah ich dieselben vollgepfropf von Akten.

Ein System für die Ordnung des historischen Materials hatte ich mir damals noch nicht zurechtgelegt. Es galt zunächst, eine Uebersicht des Vorhandenen zu gewinnen. Mein erstes Augenmerk wandle ich den Urkunden zu und begann die Regesten aller Dokumente, welche in Original und auf Pergament bis zum Ende des 10. Jahrhunderts sich. vorfanden, zu verfassen. Aus dem 16. Jahrhundert nahm ich nur die wichtigsten auf. Die Zahl dieser Regesten belief sich auf 1071 und ich fügte ein Personal- und Lokalregister nebst historischen Erläuterungen hinzu. Voll Begierde, meinen Namen gedruckt zu lesen, war ich schon gesonnen, diese Erstlinge meiner archivalischen Thätigkeit der Presse zu überliefern. Es war aber sehr gut, dass diese Idee nicht zur Ausführung gelangt ist. Denn die öffentliche Kritik hätte vielleicht meinen Sammelfleiss anerkannl, aber auch alle diplomatischen Schnitzer und chronologischen Irrthümer unbarmherzig dargethan. Meine Arbeit war indess keine unfruchtbare und leistete mir bei der Ausarbeitung der Stiftsgeschichte erhebliche Dienste.

Der schriftliche Verkehr mit dem Hrn. Landesarchivar v. Zahn und besonders dessen Schrift "Ueber die Ordnung der Urkunden am Archive des st. l. Joanneums in Graz." (Graz 1867) veranlassten mich, endlich auch für das Admonter Archiv einen Ordnungsplan zu' fixiren. Ich habe manchen Wink in dem erwähnten Werke berücksichtigt, z. B. die Einrichtung der Regesten, die Art der Aufbewahrung von Urkunden, muss aber gestehen, dass das von mir gewählte System meinem eigenen Kopfe entsprungen ist. Auch wird man wohl zugeben, dass ein und dasselbe System der Ordnung nicht für jedes Archiv sich praktisch bewährt und dass Quantität und Qualität der Archivalien, mitunter auch die zu Gebot stehenden finanziellen Mittel als massgebende Faktoren betrachtel werden müssen. 
Ich theilte meine Archivalien nach drei Hauptgesichtspunkten A. Nach der Oertlichkeit: Pfarren, Kirchen und Herrschaften. B. Nach Aemtern, wie Rent-, Kasten- und Küchenamt. C. Nach S a chen: Armenwesen, Sanitätsangelegenheiten, Jagd- und Fischbann. Die innerhalb dieser Hauptgruppen sich ergebenden Unterabtheilungen wurden chronologisch repertorisirt und aufgestellt.

Mir war es zwar bekannt, dass in den meisten Archiven Urkunden und Akten und bei Letzteren die buchförmigen von den ungebundenen strenge geschieden und besonders bearbeitet und geordnet werden. Auch ich war gesonnen, die Urkunden von den Akten zu sondern und für sich zu behandeln. Allein ich stiess auf Schwierigkeiten. Ich wollte nämlich einen eigenen Urkundenkasten für circa 4000 Dokumente hergestellt wissen, wie deren im landschaftlichen Archive zu Graz im Gebrauche stehen und sich als sehr handsam bewährt haben. Allein es blieb bei meinem Wunsche. Man wollte lange nicht glauben, dass noch nach dem Brande so Vieles und Werthvolles vorhanden sei; man hielt meine emsige Thätigkeit mehr für Zeitvertreib als ernste Arbeit und war der Meinung, dass bei der Unbedeutendheit der Archivsreste sich kostspielige Apparate nicht verlohnten. Ich muss aber der Wahrheit Zeugniss geben, dass man nach und nach zu einer besseren Auffassung der Sachlage gelangte, und dass meine Bestrebungen endlich gewürdigt und möglichst unterstützt worden sind.

Um nun die kostbare Zeit mit Abwarten nicht zu verlieren. beschloss ich, Urkunden und Akten gemeinschaftlich nach ihrer $\mathrm{Zu}$ sammengehörigkeit zu verzeichnen und einzureihen. Eine Ausnahme machte ich nur mit den ältesten Dokumenten, denen des 12. und 13. Jahrh., welche in einem eigenen geschlossenen und leicht transportablen Behältnisse verwahrt wurden.

Auf Grundlage der bereits gewonnenen allgemeinen Uebersicht und der fortschreitenden Detailarbeiten war ich bald in der Lage, über Rechtsverhältnisse oder auf Fragen wissenschaftlicher Natur, die an das Archiv gestellt wurden, Auskünfte zu ertheilen. Nun regte sich und wuchs das Interesse für das Archiv und meine Bemühungen. Man staunte, noch so viel gerettet zu sehen, und bemühte sich durch Zuwendung von Archivalien die junge Anstalt zu fördern, mich selbst aber dadurch zu erfreuen und zu ermuthigen. Mit Zustimmung meines Obern richtete ich ein Circulare an alle Pfarren und Gutsverwaltungen des Stiftes mit der Bitte, aus ihren 
Archiven und Registraturen Alles einzusenden, was für ihren Amtsbereich keine praktische Bedeutung mehr habe. Ich fand geneigtes Entgegenkommen und es lief Vieles und Bedeutendes ein. Auch aus den Registraturen des Rent- und Forstamtes wurde reichliches Materiale dem Archive zugeführt, so dass das ursprüngliche Kapellenarchiv um mehr als den dritten Theil an Zuwachs gewonnen hat.

Nun hatte das von mir übernommene Lokal den Uebelstand, dass es gegen Feuergefahr keine Sicherheit bol, und dass die Schränke, besonders die Schubladen, schr unzweckmässig waren. Die Archivalien mussten auf einander geschichtet werden, eine Partie beirrte die andere, und es kostete stets doppelte Arbeit, wenn etwas einzustellen oder auszuheben war. Eine Dislokation war daher angezeigl und der Wunsch nach ihr gerechtfertigt. Der Einzug in ein neues Lokal erfolgte im Juli 1875. Es war nämlich der ebenerdige Tract des südlichen Klosterflügels bislang als Pferdestall benützt worden. Man erbaute einen neuen Stall im Maierhofe und adoptirte den so gewonnenen Raum für die Canzleien. Bei dieser Gelegenheil wurde auch das Archivaschenbrödel mit einem neuen Kleide bedacht. Das Lokal, neben welchem sich auch das Arbeitscabinet des. Archivars befindet, hat nur zwei Fenster, ist aber hinreichend licht und für den Raumbedarf genügend. Das Zimmer ist gewölbt, die äussere Thüre mit Eisenblech beschlagen, die Fenster haben feste Eisengitter und sind mit Drahtnetzen verwahrt. Solche Drahtnetze waren es auch, welchen beim Stiftsbrande zum grossen Theile die Rettung der herrlichen Bibliothek zu verdanken ist.

Gleichzeitig wurden auch neue Schränke angeschafft, welche längs den Wänden stehen und auch den Mittelpfeiler bekleiden. Da der Plan durchgeführt wird, sämmtliche Archivalien (Urkunden und Akten) in Mappen unterzubringen, welche buchförmig an- und hintereinander gereiht werden, so mussten die Schränke diesem Zwecke entsprechend eingerichtet werden. Jeder Schrank besteht aus acht Abtheilungen, von denen vier rückwärts und höher angebracht sind. Es stehen stets zwei Mappenreihen hinter einander, und zwar die vordere tiefer, die hinlere höher. Die Mappen selbst haben ein mässiges Folioformat von gleicher Grösse und blauer Farbe, sind nur oben und vorn offen, mit Bändern versehen, und tragen auf dem Rücken die bezügliche Signatur und die Nummernreihe ihres Inhaltes. Der ganze jetzt verfügbare Raum ist für 2000 Mappen berechnet, es können aber seiner Zeit, wenn die Schränke durch- 
gängig um zwei Abtheilungen oder Gestelle erhöht werden, noch beilăufig 480 Mappen untergebracht werden. Das Ganze ist leicht zugänglich, erleichtert die Uebersicht und bietet einen gefälligen Anblick.

Innerhalb der oben erwähnten Haupteintheilung nach Orten, Aemtern und Sachen ergaben sich 122 Unterabtheilungen, welche wie im alten Archive mit fortlaufenden Buchstaben A, AA, AAA, Aa u. s. f. signirt wurden. Es wāre zwar, wie in den meisten Archiven, die Signatur mit arabischen Ziffern einfacher gewesen, allein eine gewisse Pietāt für das Herkömmliche, welche man einem ultraconservativen Klosterbruder nicht verargen wird, bestimmte mich, dem alten Gebrauche treu zu bleiben. Die Urkunden sind auf der Rückseite, um das Eigenthumsrecht zu kennzeichnen, mit einem eigenen Stempel versehen. Dieser zeigt in Vierpasse das Stiftswappen mit der Jahrzahl 1874, in welchem Jahre Admont das achthundertjährige Fest seines Bestandes feierte, und der Umschrift: "Archiv der Abtei Admont". Der Stempel wird so angebracht, dass er auf einer Texteszeile der Urkunde zu stehen kommt, daher eine Radirung oder wohl gar Ausschneidung desselben die Legende des Dokumentes verletzen oder gefährden müsste. Jede Urkunde liegt zusammengefaltet in einem Umschlag von Papier, welcher aussen zeigt die Signatur der Archivsabtheilung, die Nummer, das Regest, die Angabe, ob Original, Duplikat, beglaubigte oder einfache Abschrift, ob Pergament oder Papier, und die Zahl der Siegel mit Andeutung, ob angehängt, vorn oder rūckwärts aufgedrückt. Die Akten werden nach ihrer Zusammengehörigkeit in grössere oder kleinere Faszikel zusammen gebunden und jeder mit einem Zettel versehen, welcher über den Inhalt Aufschluss ertheilt. Ein oder mehrere dieser Faszikel werden dann, durch einen Unschlag von starkem Papier noch mehr geschützt, in den schon besprochenen Mappen eingetheilt.

Bezüglich der Repertorisirung der Dokumente und Akten machte ich anfangs den Missgriff, mich ganzer Bogen zu bedienen. Da ergab sich der Uebelstand, dass spätere Funde oder Einläufe nicht mehr an jener Stelle eingetragen werden konnten, wo sie der chronologischen Ordnung gemäss hätten ihren Platz finden sollen. Ich liess mir daher in der Druckerei eigene Zettel herrichten, so dass es nun leicht ist, allfällige Nachträge der Reihe von Regestenoder Repertoriumszetteln einzuschalten. 
Nag aber die Regestirung der Urkunden und die Repertorisirung der Akten noch so ausführlich über den Inhalt sich verbreiten, so gibt es immer noch cine Unzahl von Personen und Orlen, ron Begebenheiten und Verhältnissen, deren zwar in Dokumenten und Schriftstücken Erwähnung geschieht, die wir aber in den Repertorien vergeblich suchen. Diese reprāsentiren zunächst nur das Inventar des archivalischen Besitzstandes und können sich mit Einzelnheiten nicht befassen. Das Letztere ist Aufgabe der Personal-, Lokal- und Realregister, deren wohlgeordnete Archive nicht entrathen können. Ich hatte wohl früher bei Gelegenheit der Regestirung von 1071 Urkunden ein Personen- und Ortsregister angelegt, allein dieses umfasste nur die in den Regesten, nicht aber alle in den Dokumenten selbst vorkommenden Personen und Oertlichkeiten. Am nothwendigsten erschien mir ein Sachregister nach Schlagworten, z. - B. Altäre, Brücken, Türken - geordnet. Die bezüglichen, bald umfangreichen, bald gedrärgten Daten finden sich in mehreren Abtheilungen des Archivs zugleich und würden in den Repertorien dieser Abtheilungen öfter vergeblich gesucht werden. Das Sachregister, wenn es einmal abgeschlossen ist, belehrt mich in wenigen Augenblicken, wo Material, z. B. über Türkeneinfälle, zu suchen ist. Ein solches Register habe ich erst vor kurzer Zeit anzulegen begonnen und zwar in nachfolgender Einrichtung. Admonter-Archiv.

Grenzen.

Ort.

Zeit.

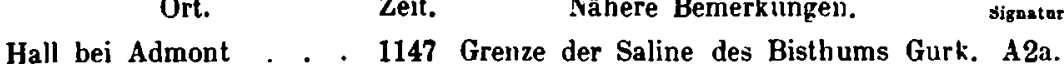
Sachregister. Freiland bei Deutschlands- 1203 Erzhischof Eherhard II. von Salzburg beberg Lungau . . . . . . 1207

Murthal .

Kärnten

Admont . . . . . . .

Admont und Gaming . . 1379 stimmt die Grenze des Gutes Freiland, A15.
A16.

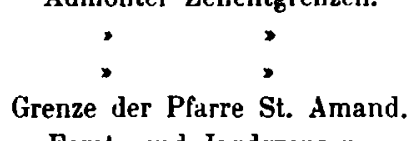

Forst- und Jagdgrenzen.

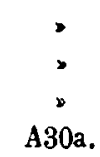

Ich habe jetzt das Archiv, wie es zur Zeit besteht, nach seinem Inhalte dem fachmännischen Leser vorzuführen.

Die ālteste Urkunde (c. 1128) ist das Diplom des Erzbischofs Conrad I. von Salzburg, in welchem er dem von ihm gegründeten Spitale zu Friesach in Kärnten Güter und Zehnten zu Hirt, Metnitz u. a. O. widmet. Das älteste von einem Admonter Abte ausgestellte Instrument datirt c. 1195, aus Weisskirchen. In 
demselben vergleicht sich Abt Rudolf II. mit Herrand von Wildon bezüglich streitiger Gutsgrenzen bei Obdach. An dieser Urkunde hängt auch das älteste Aebtesiegel, welches zugleich zu den sphragistischen Seltenheiten zăhlt. Es ist ein Doppelsiegel, die Reversseite zeigt das Siegel des Wildoners. Ist dieses als Siegel eines Gliedes des niederen Adels aus dem 12. Jahrhundert schon eine Seltenheit, so nimmt es in Verbindung mit jenem des Abtes als Doppelsiegel das höchste Interesse in Anspruch, weil solche Doppelsiegel sonst nur von Eheleuten gebraucht wurden ${ }^{1}$ ). Sonst ist von Vitgliedern des niederen Adels als ältestes Siegel jenes des Reinbrecht ron Mureck (1231) zu nennen. Das älteste Admonter Capitelsiegel hing an einer Urkunde von 1198 und hat sich an einem Dokument von 1224 erhalten. Von Admonter Aebten älterer Zeit finden sich Siegel von Gottfried II. (1223), Conrad (1237), und Albert I. (1269).

Ueberhaupt ist das Archiv ziemlich reich an alten und schönen Siegeln. Wir führen an die Bleibullen der Păpste Alexander III. (1271) und Urban IV. (1264); die Siegel des Königs Rudolf I. (1277), von welchen sechs Originalurkunden vorfindlich, der Babenberger Leopold des Glorreichen (1214) und Friedrich des Streitbaren (1241) und des Habsburgers Albrecht I. (1294). Die steiermärkischen Landesämter sind vertreten durch die Siegel des Landeshauptmannes Grafen Otto von Eberstein und des Landschreibers Witigo (1248), ferner des Landmarschalls Hartnid von Wildon (1287). An die ungarische Herrschaft erinnert das Siegel des Grafen Stefan von Agram (1257) und an die böhmische Landesoccupation jenes des Bischofs Bruno von Olmütz (1263). Von Angehörigen des höheren Adels liegen die Siegel vor von den Grafen Berthold von Andechs (1161), Friedrich von Pettau (1197), Mainhard von Görz (1232), Conrad von Wasserburg (1245), Wülfing von Stubenberg (1269) und dem Grafen Heinrich von Pfannberg (1277). Ungemein zahlreich sind die Siegel des niederen Adels. Wir erwähnen Volker von Flaschberg (1232), Erchinger von Landsee (1250) und Kalhoch von Schrattenstein (1259). Wir begegnen hier den Namen Altlengbach, Perneck, Polheim, Kapellen, Dobra, Treunstein, Ehrenburg, Erl, Falkenstein, Fulenstein, Geul, Gschiess, Hausbach, Lem-

1) Mittheil. der k. k. Centralcommission zur Erforschung und Erhaltung der Baudenkmale. XVIII. 230 und 270. 
bach, Losenstein, Ramstein, Reifenstein, Schlierbach, Stadeck, Stainach, Stretweg und Werde. Da Admont auch Beziehungen zun deutschen Ritterorden hatte, so fehlt es auch in dieser Richtung nicht an Siegeln. Wir nennen den Grossmeister Hartmann von Helderinge (1279) und die Comthure Ortolf von Traiskirchen (1236) und Conrad von Feuchtwangen (1279). Durch schöne Siegel machen sich bemerkbar die Städte Friesach (1261), Neustadt (1263), Marburg und Judenburg (1290).

Die Siegel geistlicher Würdenträger und Corporationen überwiegen an Zahl im 12. und 13. Jahrhundert alle anderen. Es seien genannt die Metropoliten von Salzburg Konrad I. (1128), Eberhard I. (1160), Konrad II. (1168), Adalbert III. (1185), Eberhard II. (1202) und Friedrich II. (1270); das Salzburger Domcapitel (1235); der Patriarch Berthold von Aquileja (1237); die Bischöfe von Freising Conrad I. (1235) und Conrad II. (1273), Heinrich von Regensburg (1283), Bernhard von Seckau (c. 1282), Dietrich II. (1261) und Hartnid (1288) von Gurk und das Domcapitel daselbst (1261), Bischof Peter von Passau (1277). An diese schliessen sich die Aebte Erchinger von Reichenbach (c. 1130), Walther von Benedictbeuren (c. 1161), Dietmar von Ossiach (1266), Ulrich von Prüfning (1283), Wernher von St. Emmeran (1283), Offo von Gleink (1284), Gottschalk von Garsten (1293), Friedrich von St. Lambrecht (1295), die Capitel der Klöster St. Peter zu Salzburg (1228), Mosach (Moggio) in Friaul (1237), der Minoriten zu Graz (1265), Prüfning (1283), Gleink (1284), Seitenstetten (1296) und Kleinmariazell (c. 1297). Hier wären noch anzureihen das Hospital am Semmering (1273) und das Admonter Nonnenkloster, das, um 1120 gestiftet, schon im zwölften Jahrhundert eine lange Reihe ausgezeichneter Frauen mit Namen aufweiset bis zu der Bibliothekarin Alheid und der Būcherabschreiberin Irmingard. An Siegeln ist dieses Kloster aber nur durch zwei des Conventes und der Meisterin repräsentirt und hängen selbe an einer Urkunde von 1327. Siegel weltlicher Frauen bis zum Ende des 14. Jahrhunderts hat das Archiv ebenfalls nur zwei aufzuweisen, nämlich jene der Giburg von Silberberg (1338) und der Herzogin Johanna von Oesterreich aus dem Hause Pfirt (1343).

Das älteste Siegel eines admontischen Beamten ist das des Propstes Heinrich von Kirchheim (c. 1236). Pröpste aber wurden die geistlichen und weltlichen Administratoren der grösseren Stiftsgüter (Propsteien) genannt. In diesem Sinne erscheinen auch Pröpstinnen 
zu St. Martin und Zeiring. Das Wappen des Stiftes (zwei Rauten neben einander) findet sich zuerst auf dem Siegel des Burggrafen von Gallenstein, Conrad von Peterdorf (1350). Auf einem àbtlichen Siegel erscheint es erst 1384. Abt Wilhelm war der Erste, welcher sein Familienwappen (von Reissberg) neben dem Schilde der Abtei auf seinem Siegel anbringen liess (1389). Das schon erwähnte Conventsiegel mit den Biltern der Heiligen Maria und Blasius im romanischen Stil war bis zum Ende des 13. Jahrhunderts im Gebrauche. Das spātere spitzovale (gothische) Capitelsiegel ist einer Urkunde von 1327 angehängt und behauptet seinen Platz bis circa 1580.

Die älteste Urkunde in deutscher Sprache datirt vom Jahr 1290, eine um 5 Jahre ältere ist verbrannt. Die erste Urkunde auf Papier ist vom J. 1361. Investiturs- und Traditionssymbole, wie Erde, Rasen, Holz u. a., welche man als Zeichen der Besitzergreifung eines Gutes zu überreichen und den Dokumenten beizulegen pflegte, sind mir nicht vorgekommen.

Die Zahl der Originalurkunden auf Pergament dürte, wenn wir die sogenannten Schirmbriefe mitzāhlen, 4000 Stücke erreichen. Davon gehören 19 dem zwölften, 125 dem dreizehnten und $206 \mathrm{dem}$ vierzehnten Jahrhundert an. Sehr gross ist auch die Ziffer der Originaldokumente auf Papier und der Abschriften aus verloren gegangenen Saalbüchern und Urkunden. Die Akten gehen bis zum Jahre 1420 hinauf.

Nun seien ganz in der Kürze die Unterabtheilungen des Archivs erwähnt.

1. Die erste Gruppe umfasst Archivalien über das Stift als s olches und dessen Centralverwaltung, wie Schutz- und Confirmationsbriefe, Privilegien, Schenkungen, Käufe, Tăusche, Verpfāndungen, beginnend mit dem Jahre 1145. Dahin gehören auch ein Bausteuerregister (15. Jahrhundert), Chronicon Admontense Amandi Pachler 1667, Regesten und Abschriften aus Saalbüchern uud Urkunden, Fragment einer Hauschronik (1659-1713), Elenchus omnium officialium monasterii, verfasst von P. Urban Ecker (1835) und gegenwärtig noch forlgeführt.

2. Beziehungen zu andern Klöstern von 1297 an. Vertreten sind die Klöster Kleinmariazell, Mondsee, Neuberg, Garsten. Dazu kommen Akten, betr. die Gesellschaft Jesu und deren Collegien zu Graz, Leoben und Ingolstadt, wobei ein Originalbrief des 
P. Wilhelm Lamormain (1630), Ossiach, St. Paul, Mönchsberg bei Bamberg, Sciz, Gaming, Spital am Pyhrn, Ottobeuern, Seon, Elchingen, Baumburg, Göttweig, Schlierbach, Thierhaupten, Herzogenburg, Arnoldstein, Lilienfeld, Vorau, Reun, die Franziskaner zu Mautern und Graz, die Kapuziner zu Irdning und die Carmeliten zu München.

3. Armenwesen. Register, Patente, Gesuche vom 16. Jahrhundert an.

4. Oblei. Kaufbriefe über zur Oblei dienstbare Güter (1318 bis 1553). Urbare und Stiftregister des 16. Jahrhunderts. Das Obleiamt war 1317 bis 1319 von dem Abte Engelbert gestiftet und hatte die Aufgabe, aus dem Erträgnisse der diesem Zwecke gewidmeten Güter die Pfründen der Klosterherren aufzubessern und besonders die nöthige Kleidung zu beschaffen. Viele Geschenke an die Oblei wurden unter der Bedingung eines Jahrtages für die Donatoren gegeben.

5. Bauten und Kunstwerke. Correspondenz mit Künstlern wie Victor Mayr und Gottfried Goetz in Augsburg, Auerbach und Bellau in Wien, und Bartholomäus Altomonte und des Letztern Handzeichnungen.

6. Bergbau. Hierbei Bergordnung König Ferdinand I. (1553) und Akten, betr. den Gagatbau in der Gams ron 1460 bis 1539, und die Bergwerke zu Johnsbach, Kallwang, Dietersdorf u. a. O. Abrisse von Hochöfen. Grubenpläne. Gagat ist eine pechartige Brennkohle. Die Gruben wurden meistens von Kaufherren aus Esslingen, Gmünd, Kirchheim, Göppingen, auch aus Nürnberg, Eschenbach betrieben. Sic mussten beim Abte um Belehnung ansuchen und den Bergzehent entrichten.

7. Forste und Weiden. Die Ur- und Hauptdotation der Admonter Abtei bestand in Grund und Boden und davon waren neun Zehntheile Waldungen. Von den beiden Klausen im Selz- und Reitthale auf beiden Seiten der Ems bis in die Frenz einerseits und von da der Salza entlang bis über Wildalpen hinaus anderseits dehnte sich ununterbrochen Waldgebirg, in welchem erst nach und nach Lichtungen entstanden, in welchen sich Kirchen und Orte (St. Gallen um 1152 und Landl 1273) erhoben. Noch jetzt ist der grösste Theil des Terrains mit Forsten bedeckt, welche zumeist an die Innerberger Actiengesellschaft, als Rechtsnachfolgerin der kk. Hauptgewerkschaft, und an die Grundholden abgetreten wurden. 
Es ist einleuchtend, dass sich in dieser Richtung ein umfangreiches Aktenmaterial gesammelt hat, welches weit in das 15. Jahrhundert hinaufreicht. Besonders wăre das Verhältniss des Stiftes, als altverbrieften Waldeigenthümers, gegenüber den Ansprüchen und Uebergriffen der Radgewerken zu Eisenerz, des landesfürstlichen Kanmergutes und der Hauptgewerkschaft ein dankbarer Gegenstand historischer Schilderung.

8. Ha mmerwerke des Stiftes zu Johnsbach, Klamm, Stegmühl und Trieben und fremder Gewerken. Urkunden vom 14. Jahrhundert an. Auf Grundlage der noch vorhandenen Urkunden lässt sich die Entstehung und Entwicklung der Eisenindustrie in den Gegenden von St. Gallen, Altenmarkt, LandI, Reifling und Lainbach sammt der Reihe der Gewerken nachweisen, aber auch darthun, dass das Kloster bemüht gewesen i=t, derlei Bestrebungen Vorschub zu leisten.

9. Nekrologien, Todtenrotel, Conföderationen. Necrologium Admontense (1731). Fragmente von Todtenbüchern (14. und 15. Jahrhundert). Die Stiftsbibliothek enthält im Codex No, 184 einen Nekrolog des XII. Jahrhunderts. Codex No. 686 hat einen solchen in mehr gedrängter Form (13. Jahrhundert); Codex No. 320 Fragmente (XII. Jahrhundert). Rotel waren um eine Rolle gewickelte schmale, aber sehr lange, auf beiden Seiten beschriebene Pergamentstreifen. Auf dem Kopfe einer solchen Encyclica wurden die Namen der zu Admont gestorbenen Mönche und Nonnen und deren Sterbtage eingetragen. Ein eigener Bote (bajulus) wurde damit in die verschiedenen Klöster mit der Bitte um deren Suffragien entsendet. Diese Klöster bemerkten dann auf der Rotel die Zeit des Eintreffens des Boten. Solcher Rotel sind noch 14 Stücke aus dem 15. Jahrhundert vorhanden. Eine davon ist von 333 Klöstern der östreichischen und deutschen Länder gefertigt. Conföderationsbriefe finden sich nur mehr in Abschriften (17. Jahrhundert). Aus diesen ergibt sich, dass Admont vom 12. bis zum 17. Jahrhundert mit folgenden Klöstern verbrüdert gewesen ist: Prüfning, Ossiach, Gleink, Lambach, St. Emmeran, Göttwejg, Melk, Seon, St. Peter, St. Lambrecht, Georgenberg (Mons Oeni, Viecht), Niederaltaich, Altenburg, Garsten, Aspach, Arnoldstein, St. Ulrich und St. Afra in Augsburg, Vorau, St. Paul, Stainz, Biburg, Fulda, Tegernsee, Rotenmann, Schlierbach, mit dem Domkapitel in Salzburg, dem deutschen Ritterorden, Orden St. Jago, der grossen Karthause und den Kapuzinern 
zu Waidhofen. - Kopialbuch ron Nekrologen des Jesuitenordens mit 406 Stücken $(1662-1677)$ und $\gg$ Litterae funebres originales societatis Jesu« (1700-1716) 399 Stücke.

10. Sakristei (Gustrei, Custodie). Urkunden betr. die zur Gustrei dienstbaren Güter von 1383 an. Urbare des 16. Jahrhunderts. Inventar des Kirchenschatzes (1659). Das Domcapitel zu Augsburg verkauft im Drange der Schwedenzeit dem Stifte A. eine silberne Ampel im Gewichle von 209 Mark (1636).

11. Sanitätswesen. Abt Johann III. und dessen Vettern Wilhelm und Hans von Trautmannsdorf machen eine Stiftung zur besseren Verpflegung der Armen im Siechenhause (1483). - Arzneibuch aus dem Beginne des 16. Jahrhunderts. Eine Masse ärztlicher Tractate und Recepte (16.-18. Jahrhundert). Pestakten.

12. Wild- und Fischbann. Auch diese Abtheilung zeichnet sich, wie jene der Forste und Weiden, durch die Fülle des Materiales aus. Den Löwenantheil behaupten die Prozesse mit den Verletzern der stiftischen Gerechtsame, Untersuchungen gegen Wildschützen; oft sehr romantischer Natur, und Fürsten- und Kaiserjagden.

13. Stiftskirche St. Blasius. Jahrtagsstiftungen des 14. und 15. Jahrhunderts. Lichtfundation von 1353. Bruderschaften. Indulgenzen. Privilegirte Altāre. Beschreibung der Kirche (16. Jahrhundert) und der Feierlichkeiten bei Uebertragung einer Reliquie der h. Erentrud (1716). Verträge mit den Goldschmieden Georg und Jacob Friedrich Guttermann (1723) und Franz Thadeus Lang zu Augsburg (1745). An Beziehungen des Klosters zu den deutschen Ländern und vorzüglich Bayern fehlte es nicht. Admont besass die Propstei Elsendorf bei Abensberg, sandte im 17. Jahrhundert seine Cleriker nach Ingolstadt oder Dillingen zu den Studien, beherbergte zur Schwedenzeit zahlreiche Flüchtlinge aus bayrischen Klöstern, und verhandelte den im Bergwerke zu Kallwang erzeugten Vitriol nach Nürnberg. Viele Glieder des Stiftes gehörten ansehnlichen Familien Deutschlands an.

14. A cta a bbatum. Familienpapiere. Reiserechnungen von 1540 an. Landtagskosten. Hochzeitseinladungen von Seite hoher Cavaliere. Empfangsbestätigung des Abtes Anton I. (1487) über einen zu Landshut gemachten Panzer. Instruction des Abtes Valentin (1552) für Kundschafter nach Nordtyrol, um sich über den Einbruch des Moritz von Sactsen durch die Ehrenbergerklause zu orientiren. Verzeichnisse von Büchern und Zeitungen, welche die 
Aebte sich bringen liessen (16. und 17. Jalırhundert). Correspondenzen und Notizbücher.

15. Nonnenkloster O. S. B. zu Admont. Urkunden rom 13. Jahrhundert an. Ablassbriefe ron 1448. 1453. 1475. Urbare der Frauenkammer (16. Jahrhundert). Zwei Professurkunden ron 1501. An Professbriefen des Herrenstiftes gingen 2-300, welche in einer Kiste verschlossen hinter dem Hauptaltare der Stiftskirche aufbewahrt wurden, beim Brande zu Grunde. Dissertatio super vitam inclitae monialis Sophiae, principis Hungaricae (17. Jahrhundert).

16. Nonnenk loster O.S. B. zu Goess bei Leoben. Urkunde von 1420. Akten über Intervention der Admonter Aebte bei Wahlen und Inventuren der Aebtissinnen (1576. 1596. 1645) und betr. die Einkleidung und Profess der Nonnen (1643-1687). Biographie der nachmaligen Aebtissin Maria Henrika Freiin ron Poppen, verfasst 1748 von dem Admonter P. Bernhard Starch. Von 1645 bis 1782 fungirte stets zu Goess ein Admonter Stiftspriester unter dem Titel "Supremus als Beichtrater und oft auch als Oeconom und Rentmeister. Einer derselbẹn, P. Marcellin Breimann, hat ein handschriftliches Chronicon Goessense (c. 1650) hinterlassen.

17. Andere Nonnenklöster, nämlich die Benediktinerinnen zu St. Georgen am Lāngsee, Nonnberg und San Cipriano zu Triest und die Dominikanerinnen zu Graz und Studenitz, anbei Visitation dieses Klosters durch Abt Raimund 1673. Briefe der Nonne Katharina von Erdödi geb. Bathyani.

18. Stift St. Lambrecht in Obersteiermark. Urkunden und Akten über den Streit zwischen Admont und St. Lambrecht bez. der Forst- und Jagdgrenzen an der Salza (14. und 15. Jahrhundert). Abtswahlen und Correspondenzen, darunter ein Brief des Admonter Abtes Valentin (1554) an den Lambrechter Abt Sigisnund, worin jener den poeta laureatus Caspar Bruschius empfiehlt, weicher » vetera monumenta librorum annalesque« zum Behufe der Drucklegung einsehen wolle. Brusch hat wirklich ein Werk des Admonter Abtes Engelbert $\gg$ De ortu et fine Romani imperii 1553 zu Basel edirt.

19. Stift St. Peter in Salzburg. So mannigfaltig auch die Beziehungen Admonts zu St. Peler gewesen sind, da Admont seine erste Ordensgemeinde von da erhalten hat, so gering ist im Archive jenes Kloster vertreten. Ausser den schon in der Abtheilung 1 befindlichen Urkunden über den Streit um das Gut Adamunta sind nur et wa zu nennen: Visitationsprotokolle (1648. 1715), verschiedene 
Hausordnungen und Verrechnungen bez. der zu Salzburg studirenden Admonter Cleriker (1634-1697).

In den Abtheilungen 20-74 folgen nun die Archivalien der Pfarren St. Nicolaus zu Rotenmann, St. Amand und St. Blasius in Admont, St. Nicolaus zu Altenmarkt, St. Anna am Lavantegg, St. Johann Bapt. in Ardning, St. Maria zu Frauenberg, H. Dreifaltigkeit zu Gaishorn, St. Gallen, St. Josef in Gams, St. Maria in Gröbming, Kirche zum h. Kreuz in Hall, St. Bartholomäus am Hohentauern, St. Egyd in Johnsbach, St. Oswald in Kall wang, St. Johann in Ka mmern, Pfarre zum h. Kreuz in Kleinsölk, St. Georg in Kra ubat, St. Bartholomäus zu Landl, St. Lorenzen im Paltenthale, St. Martin an der Salza, St. Nicolaus in Mautern, St. Michael an der Liesing, St. Andreas zu Oeblarn, St. Oswald in Freiland, Allerheiligen in Palfau, St. Nicolaus in Sausal, St. Nicolaus in Traboch, St. Cunegund in Wald, St. Cosmas und Damian zu Weng, St. Barbara zu Wildalpen, Altötting bei 0 berwölz, St. Egyden und St. Georgen in Windischbüheln, St. Georgen an der Pessnitz, St. Johann Bapt. zu Haus, St. Peter und Paul zu Irdning mit Donnersbach, St. Jacob in Freiland, St. Jacob in Leoben, St. Jacob in Windischbüheln, St. Maria in Jaring, St. Johann am Pressen, St. Johann und Paul bei Graz, Unter-St. Cunegund in Windischbüheln, St. Jacob in Lassing, St. Leonhard in Wind isch bühe In, St. Veit in Liezen, St. Maria in Oppenberg, St. Maria in Pöls, St. Maria am Rehkogel, St. Achaz zu Schladming mit Pichel und Ramsau, Pürgg, Tauplitz und Mitterndorf, St. Rupert in Trofaiach, St. Andreas zu Witschein. Diese Akten und Urkunden der Pfarren verbreiten sich wie gewöhnlich über den Erwerb von Gütern, Einkünften, Messstiftungen, Ablässen und dergleichen, enthalten mitunter Verleihungsbriefe selbst aus dem 12. Jahrhundert, Akten über Reformation und Antireformation, Weisthümer, Urbarien, Kriegs-, Mord- und Nothkalender, und eine Menge Briefe über Landanbau, Wehrbauten an Flūssen, Gewerk und Handel, sowie über die historischen Ereignisse der Umgegend.

75. Markt Admont. 1443 König Friedrich IlI. bewilligt auf Ansuchen des Abtes Andreas dem Orte A. einen ewigen Wochenmarkt. 1565 Erzherzog Karl verleiht dem Admonter Bürger Peter Seibold das Privileg des Speikhandels in Steiermark. Speik (Valeriana celtica) war damals und noch später ein stark in den Orient 
gehender Artikel. Schon die Römer kannten diese Pflanze der norischen Alpen als Mittel gegen die Kleidermotte und durch angenehmen Duft sich zur Răucherung empfehlend. Vestibus interponi eam gratissimum: Plin. histor. nat. lib. XXI. c. 20. - Salzhandelsvertrag zwischen den Orten Admont, St. Gallen und Altenmarkt (1660). Feuerlöschordnung (1762).

76. 109. 114. Weingärten überhaupt, und jene zu Luttenberg und Radkersburg insbesonders. - 1290 Ulrich, Frieàrich und Heinrich von Stubenberg schenken dem Kloster A. das Dorf Wielitsch mit Weingärten und Bergrechten. (Urk. in deutscher Sprache.) - 1563 Erzherzog Carl verbietet dem Johann Darabos, einen gewissen admontischen Weingarten mit höherem Bergrechte zu belasten. Dieser Weingarten auf der sogenannten Murinsel erscheint in Akten als sungarischer Weirgarten, und sind diesbezügliche Briefe in ungarischer Sprache (16. u. 17. Jahrh.) vorhanden.

77. 82. Gymnasien, Convicte, Schulwesen überhaupt. Das Stift besetzte 7.4 verschiedenen Zeiten mit seinen Mitgliedern die Gymnasien zu Admont, Leoben, Judenburg und Graz.

78. Zinsregister der Herren- und Frauenkammer, der Gustrei und des Amtes zum h. Geist. (1423-1579.)

79. Adels- und Wappenbriefe, Diplome gelehrter und gemeinnūtziger Vereine, Geburts-, Trauungs- und Todtenscheine, Kundschaften und Atteste verschiedener Aemter.

80. Rüstwesen und Militärsachen. Akten in das 15. Jahrh. hinaufreichend. Waffeninventare, Musterungsregister, Büchsenmeisterund Waffenschmiedrechnungen, Bestallungsbriefe über Wartgelder und Rüstpferde, Register über Landsknechtsteuer und Türkenhilfe, Befestigungsbauten zu Gallenstein, in der Frenz und Mandling, Akten über Truppentransporte und Verpflegung, französische Hilfsvölker, Reichstruppen u. dgl.

81. Documenta mixta. Hier sind Urkunden untergebracht, welche nach ihrem Inhalte und Beziehungen sich nicht leicht in eine der anderen Abtheilungen einreihen lassen, wie z. B. um 1253 befiehlt der Card.-Legat Hugo allen Kirchenvorstehern zu Ehren des von Gregor IX. heilig gesprochenen Dominicus dessen Fest feierlich zu begehen, seinen Namen den Kalendarien, Martyrologien und der Litanei einzuschalten und das officium proprium $\mathrm{zu}$ beten.

83. Handschriften. Fragmente vom 12. Jahrh. an und von Buch- und Aktendeckeln abgelöst. DeutscheSegenformel (15.Jahrh.) 
Beschreibung der Vorgänge bei der Wahl Maximilians II. zum röm. König. (1562. Gleichzeitig.) Anklageschrift gegen Albrecht von Rosenberg wegen Injurien gegen Bischof Friedrich ron Würzburg und Herzog August von Sachsen mit Beziehung auf die Grumbachschen Händel (1565). Manuscripte historischen, poetischen und ascetischen Inhalts von Admonter Kapitularen.

Dieser Abtheilung gehört auch eine Sammlung ron Autographen an, welche bis in das 16. Jahrh. hinanreicht. Wir heben hervor: Vollmacht und Instruktion des Kaisers Ferdinand II. (1632, 12. April, Wien) für den Fürsten Johann Ulrich von Eggenberg, mit Wallenstein wegen Wiederübernahme des Commandos über die kaiserl. Truppen zu unterhandeln. - Brief Jean Paul Richter's an seine Braut Karolina Mayer (1800).

84. Copien von Majestätsbriefen und Privilegien, zum Theile von Behörden vidimirt.

85. Innungen und $\mathrm{Zünfte.} \mathrm{Handwerkerordnungen} \mathrm{(z.} \mathrm{B.}$ die der Leinweber zu Graz 1509), Aufdingbriefe, Lehr- und Meisterbriefe, Streitigkeiten.

86. Correspondenzen der Kapitularen. Darunter bei 800 Briefe des steiermārkischen Historikers Albert von Muchar an seinen Freund Benno Kreil (1809-1849).

87. U rbare, Grundbücher, Güter- und Gültenverzeichnisse. In dieser Abtheilung sind nur die ältesten derselben angeführt. Urb. des Spitales in A., des Klosters und der Frauenkammer (Perg. c. 1330), des Amtes Obdach (Perg. Ende des 15. Jahrh.), der Güter in Salzburg (Perg. c. 1330, deutsch), der Güter in Oestreich (Perg. 1393-1403), des Amtes zum h. Geist (Perg 1412), des oberen Ennsthales (1424), Prachturbar über sāmmtliche Güter in zwei grossen Foliobänden, Perg. mit je 400 Blatt (1434), Urb. von 1448 (Perg. deutsch), Urbar der Probstei Sagritz aus der zweiten Hālfte des 14. Jahrh. Sechs Handurbare für den Reisegebrauch der Aebte (1617, Taschenformat).

88. Kastenamt. Dieses befasst sich zunächst mit der Aufsicht über den Getreidekasten, hat aber auch die Leitung der Mühle, Bäckerei, des Gartens und der Salzregie zu besorgen. Kastenrechnungen von 1435 an. Kaiser Ferdinand III. bewilligt dem Abte Urban und allen seinen Nachfolgern den unentgeltlichen Bezug von 200 Fudern Salz aus der Saline zu Áussee (1648). Dieser Bezug ist wohl zu unterscheiden von jenem des sogenannten Gottesheil- 
salzes, welches vielen Klöstern aus landesfürstlicher Gnade ertheilt wurde. Admont hatle seine eigenthümlichen und seit dem 12. Jahrh. selbstbetriebenen Salzwerke zu Hall und Weissenbach dem Staate abgetreten und daher ex titulo juris eine Entschädigung anzusprechen.

89. Rentamt. Rechnungen des 15. und 16. Jahrh.

90. Kelleramt. Weinleseregister von 1519 an.

91. Küchenamt. Küchendienstregister, Rechnungen, Speisebücher vom 16. Jahrh. an.

92. S chaff ner a $\mathrm{mt}$. Umfasst alle Agenden des Landbaues, der Viehzucht (Gestūte), die Direction der Handwerker und Dienstboten, das Vestiariat und das Alpenschloss Kaiserau.

93. Hofgericht. Ehemaliges Dominium Admont. Steueranschlag (1462). Institutio officii in valle Paltina (1427), in Johnsbach (1452). Weichsteuerregister (1467), auch Infel- oder Wahlsteuer genannt. Türkenhilfe (1522). Judensteuer (1497). Schirmbriefe, Inventare, Kauf- und Schuldensachen. Akten über die herrschaftliche Berainung, über Weg- und Brückenbau, Justizpflege rom 15. Jahrh. an.

94. Admontbüchel (Propstei Obdach). c. 1195 Abt Rudolf II. und Herrant von Wildon vergleichen sich über strittige Waldgrenzen bei Obdach (1 Doppelsgl.). 1214 Herzog Leopold der Glorreiche und Erzbischof Eberhard II. schlichten einen Streit zwischen A. und Reinbert von Mureck um Zehente zu Gamnar (2 Sgl., davon eines ein Doppelsgl.). Urkunden des 13. - 16. Jahrh. Steuerregister (1463). Erhebung der 1480 durch die Türken, und 1486 durch die Ungarn den Unterthanen zugefügten Schäden. Verhörsprotokoll über Zauberei.

95. Amt Bruck an der Mur mit dem Mürzthale. 1401. Revers des Jörg Herzog, dass er dem Niklas Zers sim erbern vechten das rechte Ohrläppchen abgehauen habe. Urkunden des 13.-16. Jahrh. Akten von 1476 an.

96. Admonterhof zu Graz. Urkunden des 14. und 15. und Akten des 16. Jahrh.

97. Gallenstein. Urkunden vom 14. Jabrh., Rechnungen von 1484 an. Fragment eines Urbars des 14. Jahrh. Urbar von 1424. Justizakten von 1521 an, sehr reichhaltig. Banntaidingsverhandlungen 1530-1568. Gerichtsprotokolle 1580-1771. Correspondenzen von 1546 an. Die Abtheilung Gallenstein ist eine der umfangreichsten, sie enthält 116 Faszikel. Die folgenden Abtheilungen 98-100, 103-107 harren noch der Bearbeitung. 
98. Gistadt im oberen Ennsthale. Urkunden des 13. Jahrh., zahlreicher im 14. und 15. Jahrh. Dokumente von 14507 und 1450 mit Beziehung auf Aencas Silvius, der Commendatar-Pfarrer zu Irdning war.

99. Jahringh of nordöstlich von Marburg. Urkunden und Akten des 14.-16. Jahrh.

100. Propstei Kammern. Nur neueres Material.

103. Mainhardsdorf bei Oberwölz. Aelteste Urk. von 1235 der Markgräfin Sophia von Istrien, geborene Gräfin von Andechs. 1264 Bischof Conrad II. von Freising befiehlt seinem Amtmanne zu Oberwölz, die Mauth- und Marktfreiheit der Abtei A. zu respektiren. 56 Urkunden des 14. und 15. Jahrh.

104. Marburg, Razerh of. Zwei Urkunden von 1279 betr. den Ankauf von Gütern des deutschen Ritterordens bei Marburg. 1477 Befehl Kaiser Friedrichs III. an Abt Johann von A., dem Ludwig Hausner, weil derselbe $\gg$ mit veratrey, mit inlassen vnser veint in vnser stat Furstenvelt wider vns gehandelt «, den Razerhof wieder abzunehmen (1 Sgl.).

105. St. Martin bei Graz. Urkunden von 1284 an.

106. Propstei Mautern. Urkunden des 14.-16. Jahrh.

107. Strechau bei Rotenmann mit Thalhof und Grafeneck. 1279. Wülfing von Treunstein stellt dem Stifte einen Hof bei Strechau zurück. Zahlreiche Urkunden des 15. und 16. Jahrh., welche die anwachsende Hausmacht der Hofmanne zu Grünbüchl und Strechau illustriren. Die Hofmanne mussten in Folge der Gegenreformation auswandern.

108. Zeiring mit den Gütern im Murthale. 1204. Erzbischof Eberhard II. verbrieft die von Biemul, der Witwe des salzb. Castellans Meingot, dem Stifte gemachte Donation von zwei Höfen zu Weng.

110. Güter in Bayern. Amt Elsendórf. Der einst so grossartige Güterbesitz der Abtei in Kärnten, Oesterreich und Salzburg ging schon in 16. Jahrh, verloren. Seit der furchtbare Brand 1865 das Stift zerstört hat, gehört der sReichthum des Klosters der Geschichte an und man darf auch von Wohlhabenheit nicht mehr sprechen. c. 1161 Walther, Abt von Benediktbeuren, verzichtet auf Güter zu Elsendorf. (Sglfragment). - 1161 Berthold, Graf von Andechs, beurkundet als Vogt diese Güterabtretung (1 Sgl.). 1332 Alhart der Süss, Bürger zu Regensburg, verkauft dem Abte Ekhard einen Hof 
zu Elsendorf (1 Sgl.). - 1386 Stefan, Pfalzgraf bei Rhein, und Herzog in Baiern, befreiet die admontischen Güter zu Sauerlach von jeder Vogtei (1 Sgl.). 1560 Abt Valentin und Convent verkaufen gegen Wiederkauf dem Herzog Albrecht ron Bayern das Amt Elsendorf. Akten von 1431 bis in das 18. Jahrh.

111. Kärnten. Admont besass dort die Propsteien Sagritz (Grosskirchheim) und Reichenau nebst den Aemtern Pisweg, Althofen, Krapffeld und St. Johann am Zosen. Urkunden vom 12. bis in das 16. Jahrhundert. Viele Dokumente ausgestellt von den Grafen von Görz. Akten und Urbare vom 15. Jahrhundert an.

112. Oesterreich ob und unter der Enns. Verwaltung (Hofneisterei) zu Krems mit den Aemtern Würflach, Arnsdorf, St. Peter in der Au, Winden, Hofen an der Bielach, Welbling, Brunn, Razenberg, Weinzirl und Kirchdorf. Urkunden der österr. Fürsten von 1224 an.

113. Salzburg. Propstei Fritz bei Radstadt mit dem Amte Lungau und zwei Häusern in der Stadt Salzburg. Sieben Urkunden des 13. Jahrhunderts $u$. s. w. Eine Sammlung von Formularien und ausgefertigten Urkunden. (1550-1576).

115. Acta capituli. Protokolle über Capitelbeschlüsse erst in diesem Jahrhundert beginnend. Wie weit die älteren (verbrannten) zurückreichten, ist unbekannt. Innere Angelegenheiten, Anstellungen, Correspondenzen vom 16. Jahrhundert an. Handschriftliche und gedruckte Mitgliederverzeichnisse.

116. Ecclesiastica. Akten der Dekanate verschiedene Pfarren und Kirchen betreffend. Ueberhaupt Sachen kirchenrechtlicher und liturgischer Natur.

117. Miscellanea. Da mit Miscellaneen weder einem Archivar noch einem Geschichtsforscher geholfen ist, weil die Mühe des Auffindens und der Zeitverlust in Anschlag zu bringen sind, muss diese Abtheilung nur als eine provisorisch bestehende betrachtet werden, bis für das Einzelne eine anderwärtige passende Einreihung Platz greifen kann. Einstweilen finden sich hier ein Chronicon Admontense von Amand Pachler (166i), ein Formel- und Copialbuch (Ende des 15. Jahrhunderts), Confin- und Riegbuch des Gotteshauses A. (16. Jahrhundert), Adressbuch (17. Jahrhundert), Tagebuch des P. Urban Ecker (1820-1840).

118-121. In diesen Abtheilungen sind untergebracht: eine Sammlung von Siegelabdrücken mit 4392 Stūck in 32 Kartons sammt 
Register, die Abgüsse in Schwefel, Gips und Thon, 150 Typare nicht über das 17. Jahrhundert hinaufreichend, Bleiplatten aus der Aebtegruft, Kupferstiche, Handzeichnungen, Risse und Pläne, Kupfertafeln zu Vischers steiermärk. Schlösserbuche, eine Zeitungssammlung mit 513 Stück, und eine kleine archivalische Hilfsbibliothek. In neuester Zeit (Jänner 1878) wurde auch die stiftische Mürızensammlung dem Archive einverleibt. Zur äusseren Ausschınückung des Lokales dienen alte Gemälde (Porträte der Aebte, Schlösser und Burgen), Grabsteine, Masswerke des alten gothischen Kirchenbaues zu A. (13. Jahrhundert), Waffen und Holzsculpturen.

Aus diesem in allgemeinen Umrissen entworfenen Bilde des Klosterarchives zu Admont ergibt sich die Thatsache, dass guter Wille und beharrlicher Fleiss im Stande waren, aus Asche und Trümmern ein neues, wenn auch bescheidenes, aber immerhin beachtenswerthes Gebäude zu erheben, welches vielleicht würdig gefunden wird, eine Seitenkapelle in dem erhabenen Tempel historisch-archivalischer Wissenschaft respräsentiren zu dürfen. Mag man auch die urwüchsige Art und Weise meines Vorgehens und Ordnens nicht überall billigen, so darf ich doch überzeugt sein, dass alle Freunde der Geschichte und ihrer ehrwürdigen Denkmale mein Rettungswerk, und bestände dieses auch nur in der sicheren Aufbewahrung der Pergamente und Papiere, aufmunternd begrüssen werden. 